Analele Universităţii de Vest, Timişoara

Seria Matematică - Informatică

LII, 1, (2014), 17- 27

\title{
On Exponential Trichotomy of Cocycles over Semiflows
}

Larisa Biriş and Raluca Retezan

\begin{abstract}
The paper considers a concept of exponential trichotomy for cocycles over semiflows in Banach spaces and as a particular case the corresponding dichotomy concept. Our main objective is to give a characterization of exponential trichotomy for a cocycle over a semiflow in terms of exponential dichotomy of two associated cocycles over the same semiflow.
\end{abstract}

AMS Subject Classification (2000). 34D05, 34D09

Keywords. Cocycle over a semiflow, exponential trichotomy, exponential dichotomy

\section{Introduction}

One of the most important asymptotic properties for the solutions of evolution equations is the exponential trichotomy, studied in the last years from various perspectives.

The main idea in the definition of the trichotomy property for evolution equations is to obtain at any moment, the decomposition of the state space into three closed subspaces: the stable subspace, the unstable subspace and the neutral one. In the particular case when all neutral subspaces contain only the null vector, one obtains the dichotomy property.

The notion of a cocycle over a semiflow comes naturally when one considers the linearization along an invariant manifold of a dynamical system (see for 
example [2], Chapter 6 and [10]).

Important results in the study of asymptotical behaviors of the systems described by cocycles have been obtained by S. N. Chow and H. Leiva ([3], [4]). The concept of trichotomy was identified for the first time in literature in the famous work of R. J. Sacker and J. R. Sell in [9]. They described trichotomy of linear differential systems by linear skew-product flows.

The first studies devoted to the trichotomy of differential equations were initiated by S. E. Elaydi and O. Hajek in [5].

In the last years, a substantial part of the asymptotic theory of dynamical systems was devoted to the extension of the methods used in dichotomy theory to the trichotomy case.

Characterizations of the exponential trichotomy for linear skew-product semiflows were obtained by M. Megan, C. Stoica, L. Buliga in [7] and C. Stoica and M. Megan in [13]. The case of nonuniform exponential trichotomy was considered by L. Barreira and C. Valls in [1].

A new perspective on the input-output techniques in the trichotomic behaviors of nonautonomous dynamical systems is considered by B. Sasu and L. Sasu in [11].

This paper considers a concept of exponential trichotomy for a cocycle over a semiflow and as a particular case the corresponding dichotomy concept. It is proved that the exponential trichotomy property of a cocycle over a semiflow is equivalent with exponential dichotomy of two associated cocycles over the same semiflow.

\section{Cocycles over semiflows}

Let us denote by $X$ a metric space, by $V$ a Banach space and by $\mathcal{B}(V)$ the Banach algebra of all bounded linear operators on $V$. The norm on $V$ and on $\mathcal{B}(V)$ will be denoted by $\|\cdot\|$. Let $I$ be the identity operator on $V$ and we also shall denote by $\mathbb{R}_{+}$the set of nonnegative real numbers and $Y=X \times V$.

Definition 2.1. A mapping $\varphi: \mathbb{R}_{+} \times X \rightarrow X$ is called a semiflow on $X$ if $\left(s_{1}\right) \varphi(0, x)=x$, for each $x \in X$;

$\left(s_{2}\right) \varphi(t, \varphi(s, x))=\varphi(t+s, x)$, for all $(t, s, x) \in \mathbb{R}_{+}^{2} \times X$.

A trivial example of a semiflow is given in

Example 2.1. For every metric space $X$, the mapping

$$
\varphi_{0}: \mathbb{R}_{+} \times X \rightarrow X \quad \text { defined by } \varphi_{0}(t, x)=x,
$$

for all $(t, x) \in \mathbb{R}_{+} \times X$, is a semiflow on $X$. 
Example 2.2. If $X=\mathbb{R}_{+}$(endowed with the euclidian metric) then

$$
\varphi: \mathbb{R}_{+} \times X \rightarrow X, \quad \varphi(t, x)=t+x
$$

is a semiflow on $\mathbb{R}_{+}$.

Example 2.3. Let $C$ be the metric space of all continuous functions $f$ : $\mathbb{R}_{+} \rightarrow \mathbb{R}_{+}$endowed with the topology of uniform convergence on compact subsets of $\mathbb{R}_{+}$and let $u: \mathbb{R}_{+} \rightarrow[1, \infty)$ be a decreasing function with the property that $\lim _{t \rightarrow \infty} u(t)=\alpha$. If $f \in C$ and $t \in \mathbb{R}_{+}$then we denote by $f_{t}$ the function

$$
f_{t}: \mathbb{R}_{+} \rightarrow \mathbb{R}_{+}, \quad f_{t}(s)=f(t+s), \quad \text { for each } s \in \mathbb{R}_{+} .
$$

If $X$ is the closure in $C$ of the set $\left\{u_{t}, t \in \mathbb{R}_{+}\right\}$then $X$ is a metric space and

$$
\varphi: \mathbb{R}_{+} \times X \rightarrow X, \quad \varphi(t, x)=x_{t}
$$

is a semiflow on $X$.

Definition 2.2. A mapping $\Phi: \mathbb{R}_{+} \times X \rightarrow \mathcal{B}(V)$ is called a cocycle over the semiflow $\varphi: \mathbb{R}_{+} \times X \rightarrow X$ on the space $Y=X \times V$ if

$\left(c_{1}\right) \Phi(0, x)=x$, for each $x \in X$ and

$\left(c_{2}\right) \Phi(t, \varphi(s, x)) \Phi(s, x)=\Phi(t+s, x)$, for all $(t, s, x) \in \mathbb{R}_{+}^{2} \times X$.

Example 2.4. If $X$ is a metric space and $S: \mathbb{R}_{+} \rightarrow \mathcal{B}(V)$ is a semigroup on the Banach space $V$ (i.e $\mathrm{S}(0)=\mathrm{I}$ and $S(t+s)=S(t) S(s)$ for all $(t, s) \in \mathbb{R}_{+}^{2}$ ) then $\Phi_{S}: \mathbb{R}_{+} \times X \rightarrow \mathcal{B}(V)$ defined by

$$
\Phi_{S}(t, x)=S(t), \quad \text { for every } \quad(t, x) \in \mathbb{R}_{+} \times X
$$

is a cocycle over every semiflow $\varphi: \mathbb{R}_{+} \times X \rightarrow X$ on the space $Y=X \times V$.

Example 2.5. Let $E: \Delta=\left\{(t, s) \in \mathbb{R}_{+}^{2}: t \geq s\right\} \rightarrow \mathcal{B}(V)$ be an evolution operator on the Banach space $V$ (i.e. $E(t, t)=I$ and $E(t, s) E\left(s, t_{0}\right)=$ $E\left(t, t_{0}\right)$, for all $\left.t \geq s \geq t_{0} \geq 0\right)$.

If $X=\mathbb{R}_{+}$then the mapping $\Phi_{E}: \mathbb{R}_{+} \times X \rightarrow \mathcal{B}(V)$ defined by

$$
\Phi_{E}(t, x)=E(t+x, x), \quad \text { for all } \quad(t, x) \in \mathbb{R}_{+}^{2} \times X
$$

is a cocycle over the semiflow $\varphi$ defined in Example 2.2 on $Y=\mathbb{R}_{+} \times V$. 
Example 2.6. Let $X$ be a locally compact metric space, $\varphi$ a semiflow on $X, V$ a Banach space and $A: X \rightarrow \mathcal{B}(V)$ a continuous mapping. If $y(t, x, v)$ is the solution of the abstract Cauchy problem

$$
\left\{\begin{array}{l}
y^{\prime}(t)=A(\varphi(t, x)) y(t), \quad t \geq 0 \\
y(0)=v
\end{array}\right.
$$

then the mapping $\Phi: \mathbb{R}_{+} \times X \rightarrow \mathcal{B}(V)$ defined by $\Phi(t, x) v=y(t, x, v)$ is a cocycle over the semiflow $\varphi$ on $Y=X \times V$.

Example 2.7. Let $X$ be the metric space and let $\varphi$ be the semiflow defined in Example 2.3. We consider the Banach space $V=\mathbb{R}^{3}$ with the norm $\left\|\left(v_{1}, v_{2}, v_{3}\right)\right\|=\left|v_{1}\right|+\left|v_{2}\right|+\left|v_{3}\right|$.

Then the mapping $\Phi: \mathbb{R}_{+} \times X \rightarrow \mathcal{B}(V)$ defined by

$$
\Phi(t, x)\left(v_{1}, v_{2}, v_{3}\right)=\left(v_{1} e^{-3 t u(0)+\int_{0}^{t} x(s) d s}, v_{2} e^{3 t u(0)-\int_{0}^{t} x(s) d s}, v_{3} e^{-t u(0)+2 \int_{0}^{t} x(s) d s}\right)
$$

is a cocycle over the semiflow $\varphi$ on $Y=X \times V$.

Example 2.8. If $\Phi: \mathbb{R}_{+} \times X \rightarrow \mathcal{B}(V)$ is a cocycle over the semiflow $\varphi$ : $\mathbb{R}_{+} \times X \rightarrow X$ on $Y=X \times V$ then for every $c \in \mathbb{R}$ the mapping

$$
\Phi_{c}: \mathbb{R}_{+} \times X \rightarrow \mathcal{B}(V), \quad \Phi_{c}(t, x)=e^{c t} \Phi(t, x)
$$

is also a cocycle over $\varphi$, which is called the c-shifted cocycle of $\Phi$.

\section{Exponential trichotomy of cocycles over semiflows}

Let $\Phi: \mathbb{R}_{+} \times X \rightarrow \mathcal{B}(V)$ be a cocycle over the semiflow $\varphi: \mathbb{R}_{+} \times X \rightarrow X$ on $Y=X \times V$.

Definition 3.1. A mapping $P: X \rightarrow \mathcal{B}(V)$ is called a family of projectors on the Banach space $V$ if

$$
P(x)^{2}=P(x), \quad \text { for all } \quad x \in X \text {. }
$$

The family of projectors $P: X \rightarrow \mathcal{B}(V)$ is called invariant for the cocycle $\Phi: \mathbb{R}_{+} \times X \rightarrow \mathcal{B}(V)$ over the semiflow $\varphi: \mathbb{R}_{+} \times X \rightarrow X$, if

$$
\Phi(t, x) P(x)=P(\varphi(t, x)) \Phi(t, x), \quad \text { for all } \quad(t, x) \in \mathbb{R}_{+} \times X .
$$


Definition 3.2. Two families of projectors $P_{1}, P_{2}: X \rightarrow \mathcal{B}(V)$ are called complementary if

$$
P_{1}(x)+P_{2}(x)=I, \quad \text { for each } \quad x \in X .
$$

Remark 3.1. If $P_{1}$ and $P_{2}$ are complementary then

$$
P_{1}(x) P_{2}(x)=P_{2}(x) P_{1}(x)=0, \quad \text { for every } \quad x \in X \text {. }
$$

Definition 3.3. Three families of projectors $P_{1}, P_{2}, P_{3}: X \rightarrow \mathcal{B}(V)$ are called supplementary, if

(i) $P_{1}(x)+P_{2}(x)+P_{3}(x)=I$

(ii) $P_{i}(x) P_{j}(x)=0$

(iii) $\left\|P_{i}(x) v+P_{j}(x) v\right\|^{2}=\left\|P_{i}(x) v\right\|^{2}+\left\|P_{j}(x) v\right\|^{2}$,

for all $(x, v) \in Y$ and all $i, j \in\{1,2,3\}$ with $i \neq j$.

Remark 3.2. If $V$ is a Hilbert space then in the above definition we have that $(i i) \Rightarrow(i i i)$.

Definition 3.4. Let $\Phi$ be a cocycle over the semiflow $\varphi$ on $Y$. We say that $\Phi$ is exponentially trichotomic, if there are three supplementary families of projectors $P_{1}, P_{2}, P_{3}: X \rightarrow \mathcal{B}(V)$ invariant for $\Phi$ and three constants $N \geq 1$, $a>0, b \in(0, a)$ such that

$\left(t_{1}\right) e^{a t}\left\|\Phi(t, x) P_{1}(x) v\right\| \leq N\left\|P_{1}(x) v\right\|$

$\left(t_{2}\right) e^{a t}\left\|P_{2}(x) v\right\| \leq N\left\|\Phi(t, x) P_{2}(x) v\right\|$

$\left(t_{3}\right)\left\|\Phi(t, x) P_{3}(x) v\right\| \leq N e^{b t}\left\|P_{3}(x) v\right\|$

$\left(t_{4}\right)\left\|P_{3}(x) v\right\| \leq N e^{b t}\left\|\Phi(t, x) P_{3}(x) v\right\|$,

for all $(t, x, v) \in \mathbb{R}_{+} \times Y$.

The families $P_{1}, P_{2}, P_{3}$ are called trichotomic projectors and the constants $a>b>0$ are called trichotomic constants.

If in the previous definition we have that $P_{3}(x)=0$ for every $x \in X$ then $\Phi$ is called exponentially dichotomic with the dichotomic projectors $P_{1}, P_{2}$ and the dichotomic constant $a>0$.

Example 3.1. Let $\Phi$ be the cocycle defined in Example 2.7 and let $P_{1}, P_{2}, P_{3}$ : $X \rightarrow \mathcal{B}(V)$ be the families of projectors defined by

$$
P_{1}(x)\left(v_{1}, v_{2}, v_{3}\right)=\left(v_{1}, 0,0\right), \quad P_{2}(x)\left(v_{1}, v_{2}, v_{3}\right)=\left(0, v_{2}, 0\right)
$$

and

$$
P_{3}(x)\left(v_{1}, v_{2}, v_{3}\right)=\left(0,0, v_{3}\right)
$$

which are supplementary and invariant for $\Phi$. Moreover, 


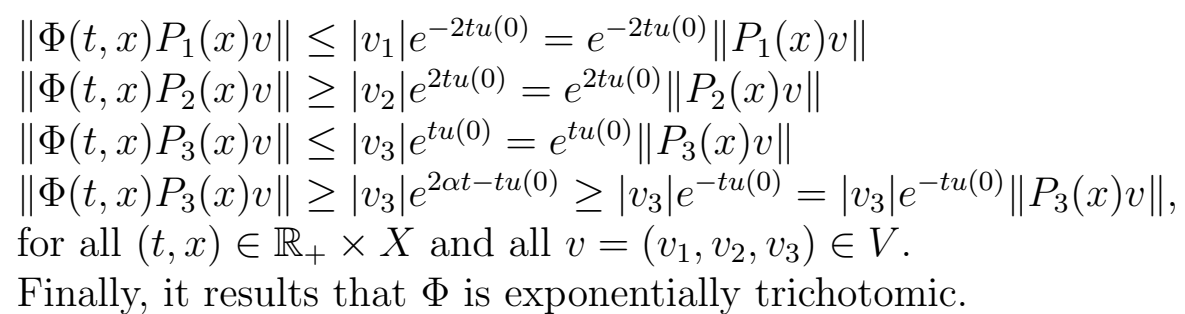

\section{Trichotomy implies dichotomy}

Let $\Phi: \mathbb{R}_{+} \times X \rightarrow \mathcal{B}(V)$ be a cocycle over the semiflow $\varphi: \mathbb{R}_{+} \times X \rightarrow X$.

Proposition 4.1. If $P_{1}, P_{2}, P_{3}: X \rightarrow \mathcal{B}(V)$ are three families of supplementary projectors and invariant for the cocycle $\Phi$ then the mappings $Q_{1}, Q_{2}, R_{1}, R_{2}: X \rightarrow \mathcal{B}(V)$ defined by

$$
Q_{1}=P_{1}, \quad Q_{2}=P_{2}+P_{3}
$$

and respectively

$$
R_{1}=P_{1}+P_{3}, \quad R_{2}=P_{2}
$$

are families of projectors with the following properties:

$\left(p_{1}\right) Q_{1}$ and $Q_{2}$ are complementary and invariant for $\Phi$

$\left(p_{2}\right) R_{1}$ and $R_{2}$ are complementary and invariant for $\Phi$

( $\left.p_{3}\right) R_{1}-Q_{1}=Q_{2}-R_{2}=R_{1} Q_{2}=Q_{2} R_{1}$

(p $\left.p_{4}\right) Q_{1} R_{2}=R_{2} Q_{1}=0$

(p $\left.p_{5}\right) Q_{1} R_{1}=R_{1} Q_{1}=Q_{1}$

$\left(p_{6}\right)\left\|R_{1}(x) v-Q_{1}(x) v\right\|^{2}=\left\|R_{1}(x) v\right\|^{2}-\left\|Q_{1}(x) v\right\|^{2}$

$\left(p_{7}\right)\left\|Q_{1}(x) v+R_{2}(x) v\right\|^{2}=\left\|Q_{1}(x) v\right\|^{2}+\left\|R_{2}(x) v\right\|^{2}$

$\left(p_{8}\right)\left\|Q_{2}(x) v-R_{2}(x) v\right\|^{2}=\left\|Q_{2}(x) v\right\|^{2}-\left\|R_{2}(x) v\right\|^{2}$, for all $(x, v) \in Y$.

Proof. It is a simple verification.

For every $c>0$ we associate to the cocycle $\Phi$ the following two cocycles $\Phi_{c}^{+}, \Phi_{c}^{-}: \mathbb{R}_{+} \times X \rightarrow \mathcal{B}(V)$ defined by

$$
\Phi_{c}^{+}=e^{c t} \Phi(t, x) \quad \text { and respectively } \quad \Phi_{c}^{-}=e^{-c t} \Phi(t, x),
$$

for all $(t, x) \in \mathbb{R}_{+} \times X$. 
Theorem 4.2. If the cocycle $\Phi: \mathbb{R}_{+} \times X \rightarrow \mathcal{B}(V)$ over the semiflow $\varphi$ : $\mathbb{R}_{+} \times X \rightarrow X$ is exponentially trichotomic with the trichotomic constants $a>b>0$ then there exists $c \in(b, a)$ such that the cocycles $\Phi_{c}^{+}$and $\Phi_{c}^{-}$are exponentially dichotomic with a dichotomic constant $d \in(0, c)$.

Proof. Suppose that $\Phi$ is exponentially trichotomic with respect to the supplementary projectors $P_{1}, P_{2}, P_{3}: X \rightarrow \mathcal{B}(V)$ and the trichotomic constants $a>b>0$.

We consider a constant $c \in\left(b, \frac{a+b}{2}\right)$. Let $Q_{1}, Q_{2}: X \rightarrow \mathcal{B}(V)$ be the families of projectors given by Proposition 4.1.

1.) We shall prove that $\Phi_{c}^{+}$is exponentially dichotomic with respect to the families of projectors $Q_{1}, Q_{2}$ and the dichotomic constant $d=c-b>0$.

$\left(d_{1}^{+}\right)$Using the condition $\left(t_{1}\right)$ from Definition 3.4 and the observation that $c+d-a<0$ we obtain that there exists $N \geq 1$ such that

$$
\begin{aligned}
& e^{d t}\left\|\Phi_{c}^{+}(t, x) Q_{1}(x) v\right\|=e^{(c+d) t}\left\|\Phi(t, x) P_{1}(x) v\right\| \leq \\
& \leq N e^{(c+d-a) t}\left\|P_{1}(x) v\right\| \leq N\left\|Q_{1}(x) v\right\|,
\end{aligned}
$$

for all $(t, x, v) \in \mathbb{R}_{+} \times Y$.

$\left(d_{2}^{+}\right)$Similarly, using the conditions $\left(t_{2}\right)$ and $\left(t_{4}\right)$ from Definition 3.4 and the invariance of $P_{2}, P_{3}$ and $Q_{2}$ for $\Phi$ it results that there is $N \geq 1$ such that

$$
\begin{aligned}
& e^{2 d t}\left\|Q_{2}(x) v\right\|^{2}=e^{2 d t}\left(\left\|P_{2}(x) v+P_{3}(x) v\right\|^{2}\right)= \\
& =e^{2 d t}\left(\left\|P_{2}(x) v\right\|^{2}+\left\|P_{3}(x) v\right\|^{2}\right) \leq \\
& \leq N^{2} e^{2 d t}\left(e^{-2 a t}\left\|\Phi(t, x) P_{2}(x) v\right\|^{2}+e^{2 b t}\left\|\Phi(t, x) P_{3}(x) v\right\|^{2}\right) \leq \\
& \leq N^{2} e^{2(b+d) t}\left(\left\|P_{2}(\varphi(t, x)) \Phi(t, x) v\right\|^{2}+\left\|P_{3}(\varphi(t, x)) \Phi(t, x) v\right\|^{2}\right)= \\
& =N^{2} e^{2(b+d) t}\left\|\left[P_{2}(\varphi(t, x))+P_{3}(\varphi(t, x))\right] \Phi(t, x) v\right\|^{2}= \\
& =N^{2} e^{2(b+d) t}\left\|Q_{2}(\varphi(t, x)) \Phi(t, x) v\right\|^{2}= \\
& =N^{2} e^{2(b+d) t}\left\|\Phi(t, x) Q_{2}(x) v\right\|^{2}= \\
& =N^{2}\left\|\Phi_{c}^{+}(t, x) Q_{2}(x) v\right\|^{2}
\end{aligned}
$$

and hence

$$
e^{d t}\left\|Q_{2}(x) v\right\| \leq N\left\|\Phi_{c}^{+}(t, x) Q_{2}(x) v\right\|
$$

for all $(t, x, v) \in \mathbb{R}_{+} \times Y$.

2.) It remains to prove that the cocycle $\Phi_{c}^{-}$is exponentially trichotomic with respect to the families of projectors $R_{1}=P_{1}+P_{3}, R_{2}=P_{2}$ and the dichotomic constant $d=c-b \in(0, c)$. 
$\left(d_{1}^{-}\right)$Similarly as in the proof of $\left(d_{2}^{+}\right)$we obtain

$$
\begin{aligned}
& \left.e^{2 d t}\left\|\Phi_{c}^{-}(t, x) R_{1}(x) v\right\|^{2}=e^{2(d-c) t} \| \Phi(t, x)\left[P_{1}(x) v+P_{3}(x) v\right)\right] \|^{2}= \\
& =e^{2(d-c) t}\left\|\left[P_{1}(\varphi(t, x))+P_{3}(\varphi(t, x))\right] \Phi(t, x) v\right\|^{2}= \\
& =e^{2(d-c) t}\left[\left\|\Phi(t, x) P_{1}(x) v\right\|^{2}+\left\|\Phi(t, x) P_{3}(x) v\right\|^{2}\right] \leq \\
& \leq N^{2} e^{2(d-c) t}\left[e^{-2 a t}\left\|P_{1}(x) v\right\|^{2}+e^{2 b t}\left\|P_{3}(x) v\right\|^{2}\right] \leq \\
& \leq N^{2} e^{2(d-c+b) t}\left[\left\|P_{1}(x) v\right\|^{2}+\left\|P_{3}(x) v\right\|^{2}\right]= \\
& =N^{2}\left\|P_{1}(x) v+P_{3}(x) v\right\|^{2}= \\
& =N^{2}\left\|R_{1}(x) v\right\|^{2}
\end{aligned}
$$

and hence

$$
e^{d t}\left\|\Phi_{c}^{-}(t, x) R_{1}(x) v\right\| \leq N\left\|R_{1}(x) v\right\|,
$$

for all $(t, x, v) \in \mathbb{R}_{+} \times Y$.

$\left(d_{2}^{-}\right)$From the definition of $R_{2}$ and the condition $\left(t_{2}\right)$ we have that there exists $N \geq 1$ with

$$
\begin{aligned}
& e^{d t}\left\|R_{2}(x) v\right\|=e^{d t}\left\|P_{2}(x) v\right\| \leq N e^{(d-a) t}\left\|\Phi(t, x) P_{2}(x) v\right\|= \\
& =N e^{(c+d-a) t}\left\|\Phi_{c}^{-}(t, x) P_{2}(x) v\right\| \leq N\left\|\Phi_{c}^{-}(t, x) R_{2}(x) v\right\|
\end{aligned}
$$

for all $(t, x, v) \in \mathbb{R}_{+} \times Y$.

\section{Dichotomy implies trichotomy}

Firstly, we prove

Proposition 5.1. Let $Q_{1}, Q_{2}, R_{1}, R_{2}: X \rightarrow \mathcal{B}(V)$ be four families of projectors which are invariant for the cocycle $\Phi: \mathbb{R}_{+} \times X \rightarrow \mathcal{B}(V)$ with the properties $\left(p_{1}\right)-\left(p_{8}\right)$ from Proposition 4.1. Then $P_{1}, P_{2}, P_{3}: X \rightarrow \mathcal{B}(V)$ defined by $P_{1}=Q_{1}, P_{2}=R_{2}$ and respectively $P_{3}=R_{1}-Q_{1}$ are three supplementary families of projectors which are invariant for $\Phi$.

Proof. It results from Proposition 4.1 using the observations

$$
P_{1}+P_{2}=Q_{1}+R_{2}, \quad P_{1}+P_{3}=R_{1} \quad \text { and } \quad P_{2}+P_{3}=Q_{2} .
$$

Let $\Phi: \mathbb{R}_{+} \times X \rightarrow \mathcal{B}(V)$ be a cocycle over the semiflow $\varphi: \mathbb{R}_{+} \times X \rightarrow X$ and let $\Phi_{c}^{+}, \Phi_{c}^{-}$be the associated cocycles defined in Example 2.8 for $c>0$. The main result of this section is 
Theorem 5.2. If there exists $c>0$ such that the cocycles $\Phi_{c}^{+}$and $\Phi_{c}^{-}$ are exponentially dichotomic with a dichotomic constant $d \in(0, c)$ then the cocycle $\Phi$ is exponentially trichotomic with the trichotomic constants $a=d+c>c-d=b>0$.

Proof. Suppose that there exists $c>0$ such that

(i) $\Phi_{c}^{+}$is exponentially dichotomic with respect to the complementary projectors $Q_{1}, Q_{2}$ and the dichotomic constant $d \in(0, c)$

and

(ii) $\Phi_{c}^{-}$is exponentially dichotomic with respect to the complementary projectors $R_{1}, R_{2}$ and the dichotomic constant $d \in(0, c)$. Consider the supplementary projectors $P_{1}, P_{2}, P_{3}$ given by Proposition 5.1. If we denote by $a=c+d$ and $b=c-d$ then $a>b>0$.

We shall prove that $\Phi$ is exponentially trichotomic with respect to the supplementary projectors $P_{1}, P_{2}, P_{3}$ and the trichotomic constants $a>b>0$.

$\left(t_{1}\right)$ By $\left(d_{1}^{+}\right)$we obtain

$$
\begin{aligned}
& e^{a t}\left\|\Phi(t, x) P_{1}(x) v\right\|=e^{(a-c) t}\left\|\Phi_{c}^{+}(t, x) Q_{1}(x) v\right\| \leq \\
& \leq N e^{(a-c-d) t}\left\|Q_{1}(x) v\right\|=N\left\|Q_{1}(x) v\right\|
\end{aligned}
$$

for all $(t, x, v) \in \mathbb{R}_{+} \times Y$.

$\left(t_{2}\right)$ By $\left(d_{2}^{+}\right)$it results that there is $N \geq 1$ with

$$
\begin{aligned}
& e^{a t}\left\|P_{2}(x) v\right\|=e^{a t}\left\|R_{2}(x) v\right\| \leq N e^{(a-d) t}\left\|\Phi_{c}^{-}(t, x) R_{2}(x) v\right\|= \\
& =N e^{(a-c-d) t}\left\|\Phi(t, x) P_{2}(x) v\right\|=N\left\|\Phi(t, x) P_{2}(x) v\right\|
\end{aligned}
$$

for all $(t, x, v) \in \mathbb{R}_{+} \times Y$.

$\left(t_{3}\right)$ Similarly, using $\left(p_{3}\right)$ and $\left(d_{1}^{-}\right)$it follows that there exists $N \geq 1$ such that

$$
\begin{aligned}
& \left\|\Phi(t, x) P_{3}(x) v\right\|=\left\|\Phi(t, x) R_{1}(x) Q_{2}(x) v\right\|= \\
& =e^{c t}\left\|\Phi_{c}^{-}(t, x) R_{1}(x) Q_{2}(x) v\right\| \leq N e^{(c-d) t}\left\|R_{1}(x) Q_{2}(x) v\right\|= \\
& =N e^{b t}\left\|P_{3}(x) v\right\|,
\end{aligned}
$$

for all $(t, x, v) \in \mathbb{R}_{+} \times Y$.

$\left(t_{4}\right)$ Because $P_{3}=Q_{2} R_{1}$, the condition $\left(d_{2}^{-}\right)$implies that there is $N \geq 1$ with

$$
\begin{aligned}
& \left\|P_{3}(x) v\right\|=\left\|Q_{2}(x) R_{1}(x) v\right\| \leq N e^{d t}\left\|\Phi_{c}^{-}(t, x) Q_{2}(x) R_{1}(x) v\right\|= \\
& =N e^{(d-c) t}\left\|\Phi(t, x) P_{3}(x) v\right\|=N e^{b t}\left\|\Phi(t, x) P_{3}(x) v\right\|,
\end{aligned}
$$

for all $(t, x, v) \in \mathbb{R}_{+} \times Y$.

Finally, we obtain that $\Phi$ is exponentially trichotomic. 


\section{The main result}

As a consequence of the Theorems 4.2 and 5.2 we obtain the main result of this paper given by

Theorem 6.1. The cocycle $\Phi: \mathbb{R}_{+} \times X \rightarrow \mathcal{B}(V)$ is exponentially trichotomic with the trichotomic constants $a>b>0$ if and only if there exist $c \in(b, a)$ and $d \in(0, c)$ such that the cocycles $\Phi_{c}^{+}$and $\Phi_{c}^{-}$are exponentially dichotomic with the dichotomic constant $d$.

In particular, for the case of a $C_{0}$ semigroup $S: \mathbb{R}_{+} \rightarrow \mathcal{B}(V)$ with the infinitesimal generator $A$, using

Definition 6.1. The operator $A$ is exponentially trichotomic, if the cocycle generated by $S$ (see Example 2.4) is exponentially trichotomic.

we obtain

Corollary 6.2. The operator $A$ is exponentially trichotomic if and only if there exists $c>0$ such that the operators $(A+c I)$ and $(A-c I)$ are exponentially dichotomic.

\section{References}

[1] L. Barreira and C. Valls, Stability of Nonautonomous Differential Equations, Springer, 1926, 2008

[2] C. Chicone and Y. Latushkin, Evolution semigroups in dynamcal systems and differential equations, Providence, R.I. Amer. Math. Soc., 70, 1999

[3] S. N. Chow and H. Leiva, Existence and roughness of the exponential dichotomy for linear skew-product semiflows in Banach spaces, J. Differential Equations, 120, (1994), 429-477

[4] S. N. Chow and H. Leiva, Two definitions of exponential dichotomy for skewproduct semiflow in Banach spaces, Proc. Amer. Math. Soc., 124, (1996), 1071-1081

[5] S. Elaydi and O. Hajek, Exponential trichotomy of differential systems, J. Math. Anal. Appl., 129, (1988), 362-374

[6] Y. Latushkin and R. Schnaubelt, Evolution semigroups, translation algebras and exponential dichotomy of cocycles, J. Differential Equations, 159, (1999), 321-369

[7] M. Megan, C. Stoica, and L. Buliga, Trichotomy for linear skew-product semiflows, Applied Analysis and Differential Equations, (2007), 227-236

[8] V. A. Pliss and G. R. Sell, Robustness of exponential dichotomies in infinitedimensional dynamical systems, J. Dynam. Differential Equations, 3, (1999), 471-513 
[9] R. J. Sacker and G. R. Sell, Existence of dichotomies and invariant splittings for differential equations, J. Differential Equations, 22, (1976), 497-522

[10] R. J. Sacker and G. R. Sell, Dichotomies for linear evolutionary equations in Banach spaces, J. Differential Equations, 113, (1994), 17-67

[11] B. Sasu and L. Sasu, Exponential trichotomy and p-admissibility for evolution equations families on the real line, Math. Z., 253, (2006), 515-536

[12] G. R. Sell and Y. You, Dynamics of Evolutionary Equations, Appl. Math. Sciences, Springer-Verlag, NY, 2002

[13] C. Stoica and M. Megan, On $(h, k)$-trichotomy for skew-evolution semiflows in Banach spaces, Studia Univ. Babes-Bolyai Math., 56, (2011), 147-156

Larisa Biriş

Department of Mathematics

Faculty of Mathematics and Computer Science

West University of Timişoara

B-dul V. Pârvan no 4

300223-Timişoara

Romania

E-mail: larisa.biris@math.uvt.ro

Raluca Retezan

Faculty of Economic Sciences

Eftimie Murgu University

Traian Vuia no 1-4

Reşiţa

Romania

E-mail: raluca.lolea@yahoo.com

Received: 29.07.2014

Accepted: 20.09.2014 